\title{
Massive Duodenal Bleeding after the Migration of Endovascular Coils into the Small Bowel
}

\author{
Chung-Jo Choi ${ }^{1}$, Hyun Lim¹, Dong-Suk Kim¹, Yong-Seol Jeong ${ }^{1}$, Sang-Young Park ${ }^{1}$ and Jeong-Eun Kim² \\ Department of ${ }^{1}$ Internal Medicine, ${ }^{2}$ Radiology, University of Hallym College of Medicine, Hallym University Sacred Heart Hospital, Anyang, \\ Korea
}

Among gastrointestinal emergencies, acute upper gastrointestinal bleeding remains a challenging clinical problem owing to significant patient morbidity and costs involved in management. Endoscopic hemostatic therapy is the mainstay of treatment and decreases the incidence of re-bleeding, the need for surgery, morbidity, and mortality. However, in $8 \%-15 \%$ of patients with upper gastrointestinal bleeding, endoscopic hemostatic therapy does not successfully control bleeding. Trans-arterial coil embolization is an effective alternative treatment for endoscopic hemostatic failure; however, this procedure can induce adverse outcomes, such as non-target vessel occlusion, vessel dissection and perforation, and coil migration. Coil migration is rare but causes severe complications, such as re-bleeding and bowel ischemia. However, in most cases, coil migration is local and involves spontaneous healing without serious complications. Here, we report the case of a patient who underwent trans-arterial coil embolization of the gastroduodenal artery with the purpose of controlling massive duodenal bleeding, resulting in a fatal outcome caused by coil migration. Clin Endosc 2019;52:612-615

Key Words: Coil migration; Endoscopy; Peptic ulcer hemorrhage; Trans-arterial embolization; Upper gastrointestinal bleeding

\section{INTRODUCTION}

Upper gastrointestinal bleeding (UGIB) is the most common gastrointestinal emergency associated with in-hospital mortality rates of over $10 \%{ }^{1}$ Endoscopy is considered crucial in the diagnosis, stratification, and management of patients with UGIB. Endoscopic hemostatic therapy reduces re-bleeding, the need for surgery, morbidity, and mortality among subjects with UGIB. ${ }^{2,3}$ However, in $8 \%-15 \%$ of patients with UGIB, endoscopic hemostatic therapy does not successfully control bleeding. ${ }^{2,3}$

Received: December 28, 2018 Revised: February 13, 2019

Accepted: February 17, 2019

Correspondence: Hyun Lim

Department of Internal Medicine, University of Hallym College of Medicine, Hallym University Sacred Heart Hospital, 22 Gwanpyeong-ro 170 beon-gil, Dongan-gu, Anyang 14068, Korea

Tel: +82-31-380-3704, Fax: +82-31-386-2269, E-mail: hlim77@hallym.or.kr ORCID: https://orcid.org/0000-0001-6581-6420

cc This is an Open Access article distributed under the terms of the Creative Commons Attribution Non-Commercial License (http://creativecommons.org/ licenses/by-nc/3.0) which permits unrestricted non-commercial use, distribution, and reproduction in any medium, provided the original work is properly cited.
When endoscopic hemostatic therapy fails to control UGIB, trans-arterial embolization (TAE) is an effective alternative treatment. ${ }^{4,5}$ Previous studies have documented the technical feasibility and high clinical success rate of TAE. ${ }^{4,5}$ The potential complications of TAE include non-target vessel occlusion, vessel dissection and perforation, and coil migration. ${ }^{4,5}$ Among these complications, coil migration is rare, but it can cause severe complications, such as re-bleeding and bowel ischemia. However, in most situations, coil migration is local and involves spontaneous healing without severe issues. ${ }^{6-8}$ Herein, we present the case of a patient with massive duodenal bleeding that occurred after the migration of endovascular coils into the small bowel and resulted in a fatal outcome.

\section{CASE REPORT}

A 65-year-old man visited the emergency department with issues such as dizziness, melena, and hematemesis. Two years previously, he was diagnosed with gallbladder cancer, and he underwent extended cholecystectomy. Emergent esophago- 
gastroduodenoscopy (EGD) showed a crater-like ulcer with massive spurting bleeding at the duodenal bulb. Endoscopic hemostasis could not be achieved. Trans-arterial angiography was urgently performed, and a significant contrast extravasation in the proximal portion of the gastroduodenal artery was observed (Fig. 1A). The active bleeding was managed with TAE (Fig. 1B). He became stable without any additional bleeding or immediate complications occurring. However, EGD performed three days after TAE indicated a duodenal ulcer and a metal object impacted at the duodenal surface (Fig. 2). Based on these findings, coil migration was highly suspected. He underwent aggressive proton pump inhibitor therapy and was discharged without adverse events 10 days after TAE. There was no change in coil position as observed on the abdominal X-ray at the time of discharge (Fig. 3). However, five days after discharge, he presented to the emergency department with massive hematemesis and cardiac arrest. Plain radiography revealed that the coil originally present in the gastroduodenal artery had migrated to the small bowel and colon (Fig. 4). Although intense resuscitative efforts were made and emergent angiography was planned, the patient eventually died.
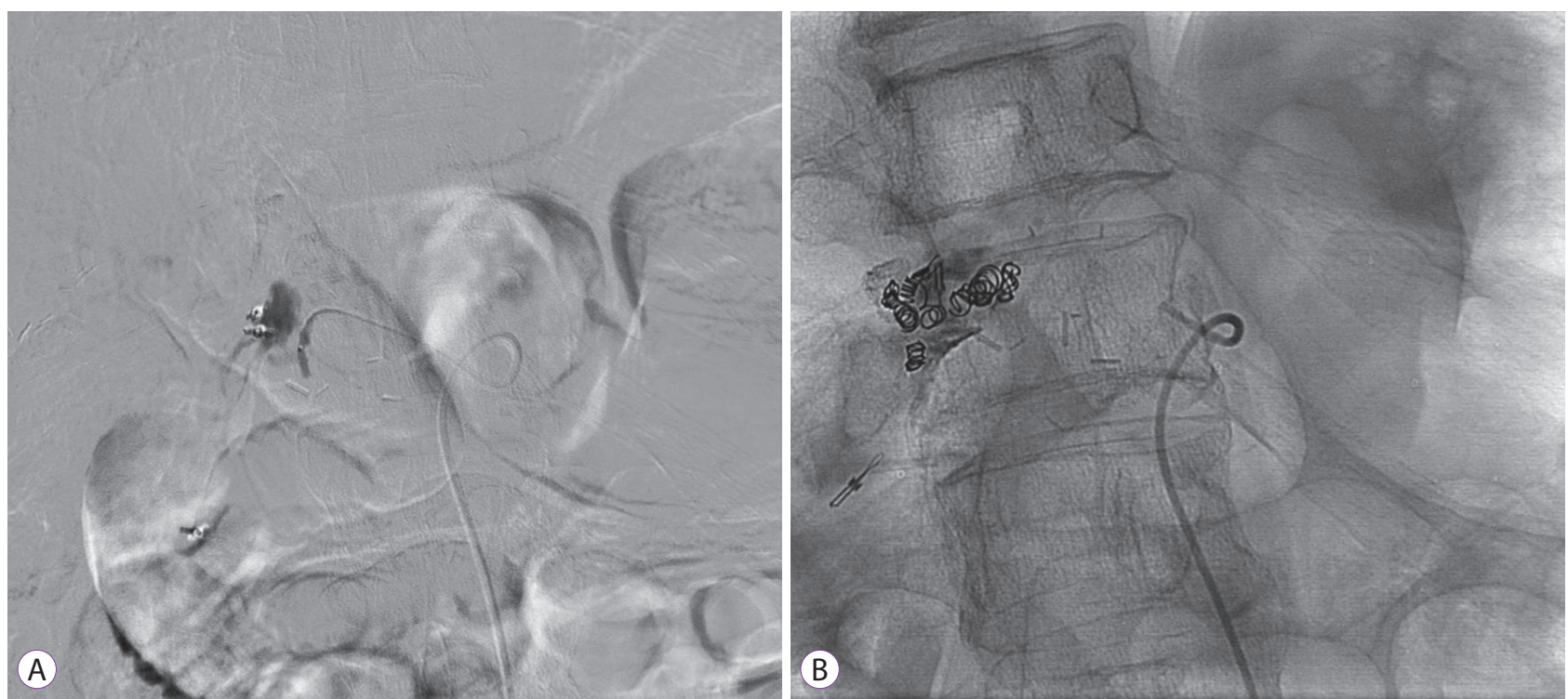

Fig. 1. Angiography image showing significant contrast extravasation at the proximal portion of the gastroduodenal artery (A). Plain radiography image showing multiple micro-coils in the successfully occluded gastroduodenal artery in the last stage of embolization (B).

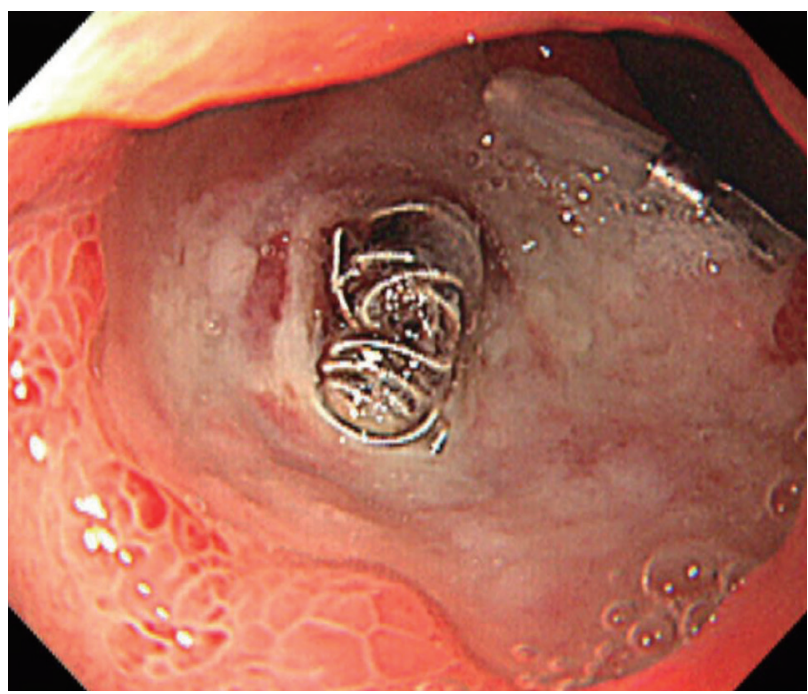

Fig. 2. Esophagogastroduodenoscopy image showing the migration of the embolized coil into the duodenum.

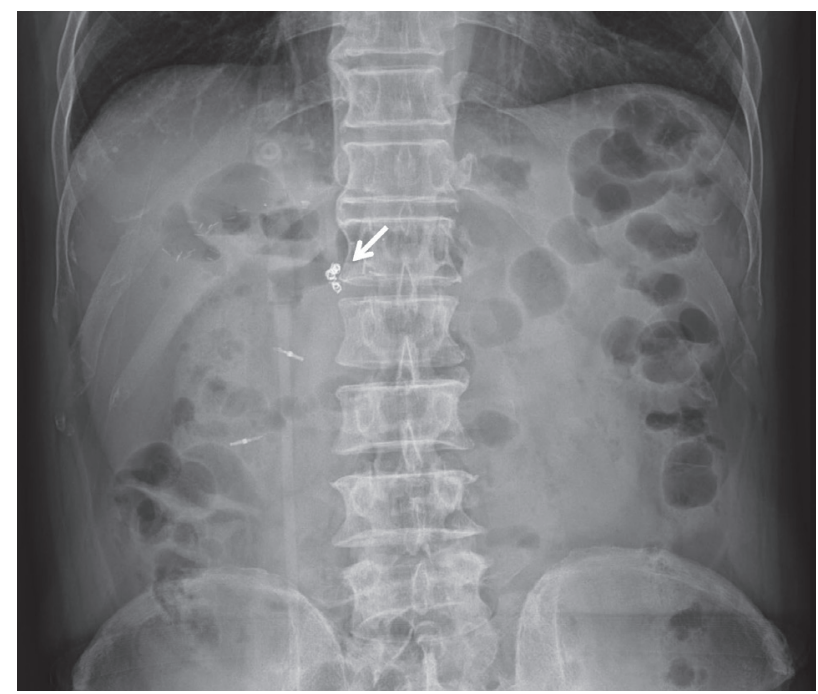

Fig. 3. Plain radiograph from the time of discharge showing no change in the position of the migrated coils (white arrow). 


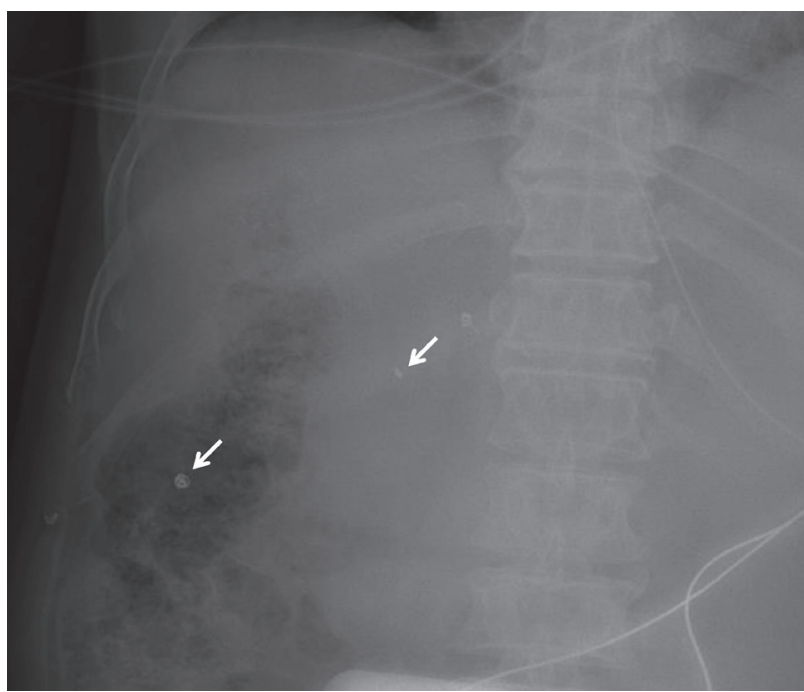

Fig. 4. Follow-up plain radiograph showings coil migration at 5 days after discharge (white arrows).

\section{DISCUSSION}

Among gastrointestinal emergencies, UGIB remains a challenging clinical problem owing to the significant patient morbidity and excessive costs involved in managing it. For UGIB, endoscopic hemostatic therapy is accepted as the firstline treatment, and several studies have confirmed that it is an effective approach to achieve hemostasis in patients with UGIB. ${ }^{2,3}$ However, endoscopic hemostatic therapy might not be effective in $8 \%-15 \%$ of UGIB cases. ${ }^{2,3}$ In patients with UGIB, the known predictors of endoscopic hemostatic failure include active bleeding with hemodynamic instability, large ulcer size, ulcer location (ulcers at the posterior bulbar duodenum and lesser curvature of the stomach are known to be associated with erosion into the gastroduodenal artery or left gastric artery), hemoglobin level, and the need for transfusion. ${ }^{9}$ Our patient presented with dizziness and hematemesis, and EGD showed an ulcer with massive spurting bleeding at the duodenal bulb. In addition, trans-arterial angiography showed significant bleeding from the gastroduodenal artery. These findings suggested that our patient had a high risk of endoscopic hemostatic failure and might need additional therapy.

Patients with a persistent bleeding despite conservative medical treatment or endoscopic hemostatic therapy require other rescue therapies, such as surgery and TAE. Surgery is usually an expeditious and acceptable approach, but it is linked to a high operative mortality rate and related to severe comorbidities. TAE is very effective at interrupting blood flow in the target vessel or lesion and stopping bleeding. ${ }^{4,5}$
Previous studies reported that the technical success rates and clinical success rates of TAE for non-variceal UGIB were 92\%-100\% and 51\%-94\%, respectively. ${ }^{4}$ In a recent European population-based cohort study, TAE was adjudged better than surgery, as it implied a shorter duration of hospital stay and fewer complications, and these benefits outweigh the high risk of re-bleeding. ${ }^{10}$ Thus, TAE is now considered the first-line therapy for massive UGIB refractory to endoscopic hemostatic therapy. In the present case, massive duodenal bleeding that could not be managed with endoscopic hemostatic therapy was successfully managed with TAE, and the patient was stable without additional bleeding or immediate complications after the procedure.

The potential complications of trans-arterial angiography and TAE include access-site hematomas (3\%-17\%) and pseudo-aneurysms, arterial dissection, ischemia (2.7\%), contrast allergic reactions, nephrotoxicity $(0.04 \%-12.7 \%)$, and coil migration. ${ }^{11,12}$ Among these, coil migration is a rare but known complication of TAE, and in some cases, coils have even passed through the rectum. ${ }^{6-8}$ The frequency of coil migration is reported to be of up to $3 \%$ among patients undergoing TAE. ${ }^{11}$ Although the precise mechanism underlying coil migration to the bowel lumen is unclear, it is known that coil embolization induces ischemia in tissues that previously received blood from vessels occluded by the coil. The damaged tissues might allow the coil to migrate partially or completely into the bowel lumen. Coil migration can cause severe complications, such as re-bleeding and bowel ischemia. However, in most instances, coil migration is local, self-limiting, and apparently non-progressive. In the present case, the embolization of the gastroduodenal artery resulted in some degree of tissue ischemia, and repeated ischemic damage led to the breakdown of the duodenal bulb and migration of the coil into the duodenal lumen. However, unlike in previous cases, in the present case, tissue ischemic damage progressed despite aggressive medical therapy, and the coil eventually completely migrated into the bowel lumen, resulting in massive bleeding and a fatal outcome.

In conclusion, we reported the case of a patient who underwent TAE of the gastroduodenal artery to control massive duodenal bleeding and who eventually died because of bleeding associated with coil migration. Although TAE is a widely used lifesaving technique in patients with UGIB refractory to endoscopic hemostatic therapy, it should be noted that adverse events, such as coil migration, could occur. In cases with coil migration, special attention should be given to the occurrence of fatal complications.

\section{Conflicts of Interest}

The authors have no financial conflicts of interest. 


\section{Author Contributions}

Conceptualization: Hyun Lim

Data curation: Dong-Suk Kim, Yong-Seol Jeong, Sang-Young Park, JeongEun Kim

Supervision: $\mathrm{HL}$

Writing-original draft: Chung-Jo Choi

Writing-review\&editing: HL, DSK, YSJ, SYP, JEK

\section{REFERENCES}

1. Hearnshaw SA, Logan RF, Lowe D, Travis SP, Murphy MF, Palmer KR. Acute upper gastrointestinal bleeding in the UK: patient characteristics, diagnoses and outcomes in the 2007 UK audit. Gut 2011;60:1327-1335.

2. Laine L, McQuaid KR. Endoscopic therapy for bleeding ulcers: an evidence-based approach based on meta-analyses of randomized controlled trials. Clin Gastroenterol Hepatol 2009;7:33-47; quiz 1-2.

3. Laine L, Jensen DM. Management of patients with ulcer bleeding. Am J Gastroenterol 2012;107:345-360; quiz 361.

4. Shin JH. Recent update of embolization of upper gastrointestinal tract bleeding. Korean J Radiol 2012;13 Suppl 1:S31-S39.
5. Loffroy R, Rao P, Ota S, De Lin M, Kwak BK, Geschwind JF. Embolization of acute nonvariceal upper gastrointestinal hemorrhage resistant to endoscopic treatment: results and predictors of recurrent bleeding. Cardiovasc Intervent Radiol 2010;33:1088-1100.

6. Singh G, Denyer M, Patel JV. Endoscopic visualization of embolization coil in a duodenal ulcer. Gastrointest Endosc 2008;67:351-352.

7. Kuhara A, Koganemaru M, Ishikawa H, Abe T. Late-occurring coil migration into the duodenum. BMJ Case Rep 2013;2013.

8. Jaurigue MM, Snyder M, Cannon M. Recurrent upper GI bleeding secondary to coil migration in a patient with known NSAID-induced peptic ulcer disease. Gastrointest Endosc 2014;79:1004 discussion 1005.

9. García-Iglesias P, Villoria A, Suarez D, et al. Meta-analysis: predictors of rebleeding after endoscopic treatment for bleeding peptic ulcer. Aliment Pharmacol Ther 2011;34:888-900.

10. Lau JY, Barkun A, Fan DM, Kuipers EJ, Yang YS, Chan FK. Challenges in the management of acute peptic ulcer bleeding. Lancet 2013;381:20332043.

11. Yap FY, Omene BO, Patel MN, et al. Transcatheter embolotherapy for gastrointestinal bleeding: a single center review of safety, efficacy, and clinical outcomes. Dig Dis Sci 2013;58:1976-1984.

12. Loffroy R, Favelier S, Pottecher P, et al. Transcatheter arterial embolization for acute nonvariceal upper gastrointestinal bleeding: indications, techniques and outcomes. Diagn Interv Imaging 2015;96:731-744. 